\title{
Pain Therapy
}

National Cancer Institute

\section{Source}

National Cancer Institute. Pain Therapy. NCI Thesaurus. Code C15180.

(i) the systematic study of clinical and basic science and its application for the reduction of pain and suffering; (ii) the blending of tools, techniques and principles taken from the discrete healing art disciplines and reformulated as a holistic application for the reduction of pain and suffering; and (iii) a newly emerging discipline emphasizing an interdisciplinary approach with a goal of reduction of pain and suffering. (AAPM) 